\title{
A Call-to-Action for Clinicians to Implement Evidence-Based Best Practices When Caring for Women with Uterine Fibroids
}

\author{
Nicholas Leyland ${ }^{1}$ (D) $\cdot$ Mathew Leonardi ${ }^{1}$ - Ally Murji ${ }^{2} \cdot$ Sukhbir S. Singh ${ }^{3} \cdot$ Ayman Al-Hendy $^{4} \cdot$ Linda Bradley $^{5}$
}

Received: 13 December 2021 / Accepted: 6 February 2022 / Published online: 17 February 2022

(c) The Author(s) 2022

\begin{abstract}
Uterine fibroids are common benign tumors that occur in up to $80 \%$ of women. Approximately half of the women affected experience considerable physical, psychological, and economic burdens and impact on quality of life due to symptoms such as heavy menstrual bleeding, pelvic pain, and infertility. Several medical and surgical options are available to treat uterine fibroids; however, healthcare providers may benefit from practical guidance in the development of individualized treatment plans based on a personalized approach. Medical treatments and minimally invasive procedures are generally preferred by most patients before considering more invasive, higher risk surgical interventions. In general, patient-centered, uterinepreserving procedures may be prioritized based on the patient's goals and the clinical scenario. Occasionally, hysterectomy may be the preferred treatment option for some patients who require definitive treatment. This call-to-action highlights recent challenges to patient care, including radical shifts in physician-patient interactions due to the COVID-19 pandemic and recent changes to evidence-based, clinically approved therapies. This report also reviews contemporary recommendations for women's health providers in the diagnosis and medical and surgical management of uterine fibroids. This call-to-action aims to empower healthcare providers to optimize the quality of care for women with uterine fibroids utilizing the best available evidence and best practices.
\end{abstract}

Keywords Abnormal uterine bleeding $\cdot$ Heavy menstrual bleeding $\cdot$ Hysterectomy $\cdot$ Leiomyoma $\cdot$ Myomectomy $\cdot$ Uterine fibroids

\section{Introduction}

Several key events took place during 2020 and the COVID19 pandemic that have impacted how physicians diagnose and treat uterine fibroids. The use of virtual medical

Nicholas Leyland

leylann@mcmaster.ca

1 Department of Obstetrics and Gynecology, McMaster University, 1280 Main St. West HSC 3 N 21, Hamilton, ON L8S 4K1, Canada

2 Department of Obstetrics and Gynecology, University of Toronto, Toronto, ON, Canada

3 Department of Obstetrics, Gynecology and Newborn Care, University of Ottawa \&, The Ottawa Hospital, Ottawa, ON, Canada

4 Department of Obstetrics and Gynecology, University of Illinois at Chicago, Chicago, IL, USA

5 Department of Obstetrics and Gynecology and the Women's Health Institute, Cleveland Clinic, Cleveland, OH, USA consultations as well as investigational and procedural delays has increased due to the COVID-19 pandemic [1]. Second, there have been significant changes to clinically available treatment options with the recent US Food and Drug Administration (FDA) approval of the first two oral treatments for uterine fibroids (elagolix with estradiol/norethindrone acetate and relugolix with estradiol/norethindrone acetate) and the withdrawal of the selective progesterone receptor modulator ulipristal acetate in Europe and Canada. Although some evidence-based guidelines [2] have been updated with guidance for the management of uterine fibroids, not all have been updated; thus, more recently available treatment options may not be reflected in existing guidelines. This call-to-action highlights current challenges and limitations to fibroid diagnosis and management and examines newer treatment options to help clinicians make updated, evidence-based decisions to treat patients with uterine fibroids. 


\section{Overview of Disease Burden}

Uterine fibroids (also known as leiomyomas or myomas) are benign, monoclonal neoplasms that form in the myometrium and are associated with symptoms such as heavy menstrual bleeding (HMB), bulk-related symptoms (e.g., pelvic pain and pressure), and reproductive dysfunction (e.g., obstetric complications and infertility) [3]. Uterine fibroids are the most common type of benign pelvic tumor in women $[4,5]$ and are associated with decreased quality of life (QOL) due to negative impacts on social and intimate relationships, physical activity, work productivity, and psychological well-being [5]. Uterine fibroids also lead to significant individual and societal economic impacts, with costs estimated at (US) \$9.4 billion per year; these costs are attributed to direct and indirect expenditures associated with obstetric complications, diagnosticand treatment-related costs, costs of sanitary products, and increased absenteeism and presenteeism [6,7]. Major risk factors for uterine fibroids include age (up to menopause) and Black race $[3,8]$. Women of color are three times more likely to develop fibroids, present at an earlier age, experience more severe symptoms, and have higher rates of hospitalization and surgical intervention (myomectomies and hysterectomies) compared with White women $[9,10]$. The disproportionate impact on Black women has wide-ranging implications stemming from known socioeconomic disparities and reduced access to healthcare [10]. As a result, uterine fibroid management has a widereaching impact on society at large.

\section{Challenges and Limitations of Uterine Fibroid Management}

\section{Challenges in Diagnosis}

Diagnosis of uterine fibroids and selection of treatment options are guided by imaging techniques such as ultrasound, sonohysterography, or magnetic resonance imaging (MRI). However, lack of adherence by physicians to standardized imaging guidelines represents a clinical challenge. In fact, findings from a recent Canadian report show that physicians do not consistently follow the recommendations outlined in the Morphological Uterus Sonographic Assessment consensus statement $[11,12]$. In addition, the quality of ultrasound reports falls short of internally endorsed guidelines and requires improvement [12]. Clear, highquality imaging is required for physicians to determine fibroid size, location, and impact on the endometrial cavity and the endometrium. Standardized quality performance, interpretation, and reporting are essential to inform diagnoses and guide physicians to counsel their patients in choosing appropriate and optimal therapies. Ultrasonography is the most commonly used imaging technique to diagnose and monitor uterine fibroids, owing to the technology's high availability, ease of use, and low cost [12]; however, inaccurate interpretation of ultrasonography images may lead to misdiagnoses [13]. Sonohysterography is also commonly used to diagnose submucosal fibroids and evaluate the degree of endometrial cavity involvement [14]. Improved imaging guidelines are needed to help physicians more accurately diagnose patients (e.g., distinguish uterine fibroids from adenomyosis or other malignancies), evaluate the uterine cavity, predict therapeutic response, or anticipate potential surgical approaches and challenges during such procedures. Beyond improving the quality of what we currently have access to, newer imaging options include strain elastography and shear wave elastography, which may help guide diagnoses and inform treatment decisions by providing information on tissue stiffness [13, 15].

In addition to improved development and adherence to imaging guidelines and accurate diagnosis, there is a need for wider adoption of these recommendations across the world. The most recent and widely used current classification system, developed in 2011 [16] and modified in 2018 [17] by the International Federation of Gynecology and Obstetrics, is based on fibroid location and degree of intramural/intracavitary extension. This classification system has been widely adopted and is easy to follow; however, the location-based system may not necessarily correlate with tumor biology or patient symptoms [18]. The lack of pathological criteria for non-surgical interventions also presents a diagnostic challenge [19]. Additional research is needed to further characterize and distinguish specific subtypes of uterine fibroids to account for various features of fibroid pathophysiology and more effectively guide individualized diagnoses and interventions.

\section{Challenges Due to the COVID-19 Pandemic and Virtual Medicine}

Physician-patient interactions have changed in recent times due to the shift from in-person consultations to virtual appointments precipitated by the COVID-19 pandemic. Although there are many apparent benefits to telehealth [20, 21], including safety, convenience, and increasing access to care, concerns have been raised around clinical practice patterns for patients in remote settings [22]. In addition, the lack of in-person assessment may limit accurate evaluation or examination of the patient and prevent or delay tissue sampling [1], leading to misdiagnoses or delayed diagnoses, delays in blood testing (e.g., to identify the degree of 
anemia), or missed identification of acute emergencies such as prolapsed fibroids. Delayed assessment, diagnosis, and intervention may also cause lesions to enlarge and symptoms to progress, negatively impacting patient QOL [23, 24]. In the case of procedural delays due to pandemic-related shifts in hospital policies or patient fears, medical options may help bridge the gap in treatment, reduce the volume of lesions, and optimize hemoglobin levels until such time that the patient is able to undergo surgery. However, surgical guidelines developed during the COVID-19 pandemic recommend that patients with urgent benign gynecological issues and substantial symptoms should not delay surgical intervention [25]. Furthermore, the COVID-19 pandemic has disproportionally affected women of color, who are more likely to contract COVID-19, are at increased risk for hospitalization and mortality due to COVID-19, have reduced access to high-quality healthcare, and are more likely to work outside the home and/or in jobs without paid sick leave compared with White women [26-29].

\section{Prevention, Diagnosis, and Treatment of Anemia}

Another current challenge in treating patients with uterine fibroids is the management of iron deficiency anemia, which is a common comorbidity experienced by approximately two-thirds of women who experience HMB with uterine fibroids [30-33]. The recommended primary prevention strategy for iron deficiency anemia is adequate intake of dietary iron [34]. Anemia can substantially impact QOL and appropriate treatments for iron deficiency are generally associated with improvements in QOL [30, 35, 36]. Oral supplementation with iron salts is commonly used as first-line therapy for the treatment of anemia; if patients do not improve or cannot tolerate iron salts, intravenous iron is recommended [37]. Once anemia is corrected it may require up to 3-6 months to replete iron stores; iron supplementation should continue after anemia resolves. In addition to oral or parenteral iron, medical management that decreases menstrual blood loss is a valuable adjunct method to treat anemia. Appropriate screening and treatment of anemia is recommended to improve symptoms and correct hemoglobin levels before surgery due to the increased risk of postoperative morbidity and mortality associated with anemia [15, 38]. Surgeons should aim for hemoglobin levels $>12.0 \mathrm{~g} / \mathrm{dL}$ prior to performing elective fibroid surgery [39]. Perioperative blood transfusions can generally be avoided in treating anemia, as transfusions are associated with significant morbidity, including sensitization leading to the potential development of hemolytic disease in the fetus and newborn [38]. The development of clinically significant alloantibodies occurs in approximately $8.1 \%$ of patients who receive blood transfusions [38]. Such sensitization is an important issue to prevent in women with fibroids who are planning future fertility. Aggressive treatment of anemia is important, especially before surgery, and can help reduce the need for blood transfusions.

\section{Management of Uterine Fibroids}

\section{Expectant Management}

Many patients with uterine fibroids do not experience any symptoms; in fact, incidental discovery of fibroids is relatively common $[31,33]$. For these patients, no treatment may be necessary, and watchful waiting may be considered [31, $40,41]$. However, it is important to tailor interventions for individual patients based on menopausal status, the patient's desire to preserve fertility, or where there is a suspicion of malignancy. Patient-centered disease management is guided by medical history, physical examination, imaging, and results of blood tests. If patients begin to experience symptoms and desire treatment, a fibroid-specific management plan can be developed as outlined in the following sections.

\section{Medical Management}

Several options for medical management are currently available to decrease uterine fibroid size, reduce menstrual blood loss, improve hemoglobin levels, and/or improve fibroidassociated symptoms (Fig. 1 and Table 1) [31, 40-42]. Not all medical management options provide contraception, but some of them (e.g., oral contraceptives) have that additional benefit. Medical treatments may be used while patients await surgery to manage fibroid size and symptoms or may even replace surgical options in some cases [31]. When given the choice between surgery and pharmaceutical management of uterine fibroids, many women would choose the latter to avoid major surgery or to address other personal preferences [43, 44]. It is important to note that not all the clinical practice guidelines have been updated to include all approved pharmaceutical options for medical management of fibroids. The most recent American College of Obstetricians and Gynecologists practice bulletin on the management of fibroids was published in 2021 [2] and a comparative effectiveness review was published in 2017 by the Agency for Healthcare Research and Quality [7]. The most recent update to the 2015 Society of Obstetricians and Gynecologists of Canada guidelines [33] for medical management of symptomatic fibroids was published in 2019 [45]. Decisions regarding the medical management of uterine fibroids should be guided by the physician's assessment, appropriately tailored for each patient, and shared between the patient and physician [2, 41, 46]. Evidence-based treatment plans must 
Fig. 1 Treatment options for uterine fibroids. Evidence-based treatment decisions should be tailored according to the individual clinical scenario (e.g., size and location of fibroids, patient age, symptoms, desire to preserve fertility, access to therapy) and clinician judgment

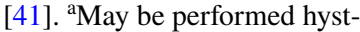
eroscopically, laparoscopically, abdominally, or with robotic assistance. GnRH, gonadotropin-releasing hormone; $\mathrm{HMB}$, heavy menstrual bleeding; MRI, magnetic resonance imaging

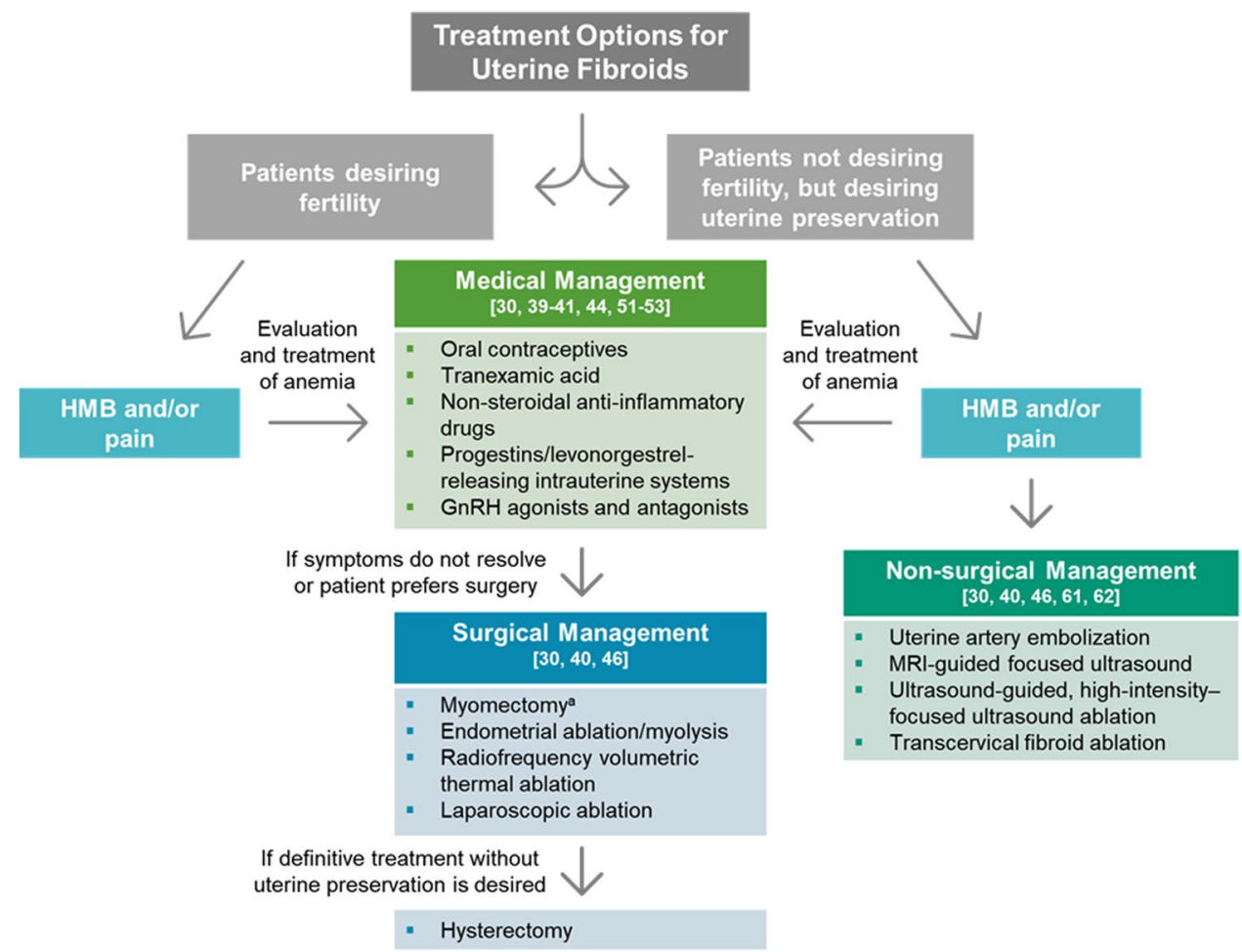

Table 1 Medical management of uterine fibroids

\begin{tabular}{|c|c|}
\hline Treatment & Evidence-based recommendation \\
\hline Oral contraceptives (estrogen/progestin) & Reduces HMB but does not inhibit fibroid growth or reduce fibroid volume [31, 40-42] \\
\hline Tranexamic acid & $\begin{array}{l}\text { Non-hormonal oral antifibrinolytic agent; reduces HMB but has no effect on fibroid size; } \\
\text { widely available globally }[31,41,42]\end{array}$ \\
\hline Non-steroidal anti-inflammatory drugs & $\begin{array}{l}\text { Reduces HMB and pain, though less effectively than estrogen/progestin contraceptives, } \\
\text { the levonorgestrel-releasing intrauterine system, or tranexamic acid }[31,41]\end{array}$ \\
\hline Oral or injectable progestins & Reduces HMB but data supporting effectiveness are limited $[2,41]$ \\
\hline Levonorgestrel-releasing intrauterine system & $\begin{array}{l}\text { Reduces HMB to a greater extent than oral contraceptives; may have limited benefits in } \\
\text { women with high fibroid burden that distorts the uterine cavity due to risk off expulsion } \\
{[31,41,42]}\end{array}$ \\
\hline GnRH agonists & $\begin{array}{l}\text { Reduces HMB, significantly reduces fibroid size, and improves hemoglobin levels; } \\
\text { recommended in combination with low-dose estrogen/progestin add-back therapy to } \\
\text { mitigate adverse effects and/or as pretreatment to reduce fibroid volume before surgery } \\
(3-6 \text { months })[7,31,40-42,53]\end{array}$ \\
\hline GnRH antagonists & $\begin{array}{l}\text { Reduces HMB and fibroid volume; improves hemoglobin levels; recommended in com- } \\
\text { bination with low-dose estrogen/progestin add-back therapy to mitigate adverse effects } \\
{[31,42]}\end{array}$ \\
\hline Selective progesterone receptor modulators & $\begin{array}{l}\text { Reduces HMB, pain, and fibroid volume and increases hemoglobin levels; recommenda- } \\
\text { tions suspended in } 2020 \text { due to safety concerns; long-term safety is under investigation } \\
{[31,42,56,57]}\end{array}$ \\
\hline Aromatase inhibitors & Limited evidence to demonstrate reductions in $\mathrm{HMB}$ or fibroid size $[31,41]$ \\
\hline Natural therapy (vitamin D, epigallocatechin gallate) & $\begin{array}{l}\text { May inhibit fibroid growth; currently under clinical investigation and further evaluation is } \\
\text { needed }[31,58,59]\end{array}$ \\
\hline
\end{tabular}

$G n R H$, gonadotropin-releasing hormone; $H M B$, heavy menstrual bleeding

consider fibroid type (size, location, and characteristics) and patient's symptoms, medical history, desire for fertility, access to therapies, and impact on QOL [31].
Currently available hormonal therapies for the treatment of uterine fibroids include combined estrogen/progestin oral contraceptives, progestin-only contraceptives (including the 
progestin-(levonorgestrel-) releasing intrauterine system [LNG-IUS]), and gonadotropin-releasing hormone (GnRH) agonists and antagonists. Combined estrogen/progestin oral contraceptives are commonly used as first-line therapy for the treatment of uterine fibroids; these agents reduce fibroidassociated symptoms such as HMB, but do not inhibit fibroid growth or reduce fibroid volume [31,40-42]. Oral or injectable progestins can reduce HMB, but they are not currently recommended to treat uterine fibroids because there is a lack of sufficient evidence to prove their effectiveness [40, 41]. Other non-hormonal options, such as the antifibrinolytic agent tranexamic acid or non-steroidal anti-inflammatory drugs, also reduce HMB and other fibroid-associated symptoms, but do not inhibit fibroid growth or reduce fibroid size [31, 40-42]. Non-steroidal anti-inflammatory drugs are less effective in reducing HMB than tranexamic acid, combined estrogen/progestin oral contraceptives, or the LNG-IUS [31, 41].

The LNG-IUS acts locally on the endometrium to decrease HMB (leading to amenorrhea or improvements in menorrhagia) and reduces HMB to a greater extent than do oral contraceptives [31, 41, 42, 47]. A systematic review of 10 studies evaluating the LNG-IUS to treat uterine fibroids demonstrated substantial reductions in HMB; some studies showed reductions in uterine and fibroid volumes, while others showed no change $[47,48]$. The LNG-IUS is indicated for long-term, reversible contraception and HMB, but is contraindicated for women with a high fibroid burden that distorts the uterine cavity. Risk of LNG-IUS expulsion is higher in women with fibroids; reported expulsion rates are between 0 and $20 \%$ [31, 41, 42].

$\mathrm{GnRH}$ antagonists are the most recent addition to the options for the treatment of uterine fibroids [40]. Elagolix, an oral GnRH receptor antagonist, administered in combination with hormonal add-back therapy (estradiol $1 \mathrm{mg}$ and norethindrone acetate $0.5 \mathrm{mg}$ once daily; E2/NETA) is the first FDA-approved (May 2020) oral treatment option for uterine fibroids and is indicated for the management of HMB associated with fibroids in premenopausal women for up to 24 months. The Elaris Uterine Fibroids 1 and 2 studies were identically designed as 6-month, phase 3 randomized trials that evaluated the efficacy and safety of elagolix with add-back therapy in women with fibroid-associated HMB. Findings from these studies indicated that patients taking elagolix with add-back therapy experienced significantly less HMB associated with uterine fibroids compared with patients taking placebo [49]. Elagolix is also highly effective in decreasing fibroid volume $[31,50]$; however, the effects of elagolix on fibroid size are diminished with add-back therapy. Another GnRH antagonist, relugolix, was approved by the FDA in May 2021 in combination with E2/NETA for the management of HMB in premenopausal women. This approval followed the recent publication of two 24-week randomized, placebo-controlled, phase 3 clinical studies that demonstrated significantly higher reductions in fibroidassociated HMB and pain, reduced uterine volume (suggesting decreased fibroid burden), and increased hemoglobin levels with relugolix and E2/NETA compared with placebo [51]. Findings from a recent phase $2 b$ study indicated that linzagolix significantly reduced endometriosis-associated pain [52], and linzagolix is currently being evaluated in clinical trials to treat HMB associated with uterine fibroids and reduce fibroid volume [42]. This class of drugs has the potential to significantly improve the management of acute and chronic HMB; however, they may be less available and/ or more expensive in countries without universal access to healthcare.

Results from recent studies have also shown that the GnRH agonists leuprolide, goserelin, and triptorelin decrease fibroid volume, reduce HMB and fibroid-associated pain, and increase hemoglobin levels [31]. A Cochrane review [53] examined findings from multiple randomized controlled trials and found clear evidence that GnRH ana$\operatorname{logs}$ (both antagonist and agonists) can improve hemoglobin levels and reduce fibroid and uterine volume. One challenge with these agents is the appropriate administration with respect to the patient's menstrual cycle to avoid a hormonal "flare" effect and associated increased HMB.

GnRH agonists and antagonists have been associated with adverse effects such as hot flushes, decreased libido, and sleep disturbances; however, many of these effects can be successfully alleviated with hormonal add-back therapy [31, 42]. Loss in bone mineral density (BMD) has also been associated with GnRH agonists and antagonists; evidence from the Elaris [49] and Liberty [51] studies demonstrated that even with add-back, BMD loss is observed and long-term implications of this are currently unknown. Addition of addback therapy does slightly reduce the beneficial effects of GnRH analogs on menstrual blood loss and fibroid volume; however, the benefits of adding add-back therapy for the management of uterine fibroids are important to consider.

Selective progesterone receptor modulators were initially considered promising treatment options and effectively reduced fibroid volume, treated symptoms, and delayed or eliminated surgical intervention [54]. However, these therapies have recently been scrutinized because of safety concerns, specifically the rare occurrence of liver dysfunction leading to liver failure $[40,55]$. In several clinical trials, ulipristal acetate has demonstrated significant reductions in fibroid volume and HMB along with improvements in hemoglobin levels and was approved in Canada and Europe for medical management of uterine fibroids [31, 42]. However, ulipristal acetate was withdrawn in Canada and Europe in 2020 after cases of drug-induced liver toxicity were reported $[42,56,57]$. In the USA, ulipristal acetate is no longer under clinical evaluation nor is FDA approval being sought for 
its use in treating symptomatic uterine fibroids. The overall risk of liver injury leading to transplantation was extremely low (six of over 1,000,000 cases), but these cases were not predictable or related to dose [42]. Other selective progesterone receptor modulators including mifepristone, asoprisnil, vilaprisan, and telapristone acetate have also demonstrated efficacy in reducing fibroid-associated symptoms in randomized controlled trials, but clinical investigation is currently paused $[31,56]$. Other agents under investigation for the medical management of uterine fibroids include cabergoline, gestrinone, somatostatin analogs, and natural compounds such as vitamin D and green tea extract (epigallocatechin gallate) $[31,58,59]$.

\section{Surgical Management}

For more than 100 years, surgical interventions (myomectomy or hysterectomy) have been the main options available to treat uterine fibroids (Table 2); however, surgical procedures may be associated with a high psychological and economic burden, and there is a risk of recurrence of uterine fibroids with uterine-sparing procedures [46]. Hysterectomy is the most common surgical management option and the only method to definitively stop fibroid-associated HMB [30, 31, 41]. Although hysterectomy is associated with risk of perioperative morbidity, in certain cases, it may be the best treatment option in patients who have completed childbearing, and/or have had multiple recurrences of uterine fibroids following other medical or surgical interventions and desire definitive treatment. If surgical management is recommended, referral to high-volume surgeons and medical centers is advised to improve outcomes, decrease length of surgery, and decrease risk of complications. Patients undergoing surgery also benefit when enhanced recovery protocols are employed to improve recovery and patient experiences.
Whenever possible, patients should be made aware of less invasive and uterine-sparing procedures, which may minimize the risk to the patient. These include myomectomy via minimally invasive techniques when appropriate for fibroid removal and improvement of bulk symptoms or other less invasive techniques such as endometrial ablation or radiofrequency volumetric thermal ablation to control bleeding $[31,60]$. Myomectomy is recommended for patients wishing to preserve fertility; hysteroscopic myomectomy is generally recommended for submucosal fibroids, while laparoscopic or laparotomic myomectomy is advised for intramural and subserosal fibroids [31].

\section{Non-surgical Management}

Other less invasive non-surgical alternatives include uterine artery embolization to interrupt blood flow to the uterus and fibroids and MRI-guided, high-intensity-focused ultrasound to induce fibroid necrosis and regression (Table 3) [31]. Ultrasound-guided, high-intensity-focused ultrasound ablation has also been shown to improve fibroid symptoms and reduce fibroid volume without permanent adverse effects [61]. These techniques are often effective for symptom control and are associated with shorter recovery times and fewer complications compared with surgery, but may not be good options for patients desiring to retain fertility [31]. Another novel minimally invasive approach not yet included in guidelines for the treatment of uterine fibroids is transcervical fibroid ablation, a uterus-preserving technique that locates fibroids using intrauterine ultrasound and treats them with radiofrequency energy [62]; this outpatient procedure has been shown to be safe and effective in multiple studies [62] but is not yet widely available. The challenge with all the aforementioned procedures is the lack of pathological diagnosis with such interventions.

Table 2 Surgical management of uterine fibroids

\begin{tabular}{|c|c|}
\hline Treatment & Evidence-based recommendation \\
\hline Hysteroscopic myomectomy & $\begin{array}{l}\text { Decreases and removes intracavitary fibroids and improves symptoms; typically preserves the } \\
\text { integrity of the myometrium; recommended for FIGO 0, FIGO 1, and some FIGO } 2 \text { submu- } \\
\text { cosal fibroids and for patients desiring to retain fertility; associated with a 15-50\% risk of } \\
\text { recurrence }[31,41,46]\end{array}$ \\
\hline $\begin{array}{l}\text { Abdominal myomectomy (laparoscopic, } \\
\text { robotic, or laparotomic) }\end{array}$ & $\begin{array}{l}\text { Reduces uterine volume and improves symptoms; recommended for intramural, subserosal, and } \\
\text { very large submucosal fibroids that are not amenable to hysteroscopic resection [31] }\end{array}$ \\
\hline Endometrial ablation/myolysis & $\begin{array}{l}\text { Reduces HMB; uses electrical energy, cryotherapy, heated saline, or radiofrequency energy to } \\
\text { destroy the endometrium; recommended for premenopausal patients who do not desire future } \\
\text { fertility }[31,41,46]\end{array}$ \\
\hline Radiofrequency volumetric thermal ablation & $\begin{array}{l}\text { Minimally invasive; reduces fibroid volume and improves symptoms; impact on fertility requires } \\
\text { further investigation [31] }\end{array}$ \\
\hline Hysterectomy & $\begin{array}{l}\text { Advised for patients who desire definitive treatment for symptomatic fibroids; should be per- } \\
\text { formed minimally invasively when possible }[31,41,46]\end{array}$ \\
\hline
\end{tabular}

FIGO, International Federation of Gynecology and Obstetrics; $H M B$, heavy menstrual bleeding 
Table 3 Non-surgical management of uterine fibroids

\begin{tabular}{ll}
\hline Treatment & Evidence-based recommendation \\
\hline Uterine artery embolization & $\begin{array}{c}\text { Minimally invasive; reduces symptoms and decreases fibroid volume by limiting blood supply to the } \\
\text { fibroids as non-involved uterus is spared; recommended for patients who are not good surgical can- } \\
\text { didates or who choose to avoid surgery; may impact uterine and ovarian function; impact on fertility }\end{array}$ \\
requires further investigation [31, 41, 46] \\
Minimally invasive yet effective for controlling symptoms and reducing fibroid size; recommended \\
for patients who are not good surgical candidates or who choose to avoid surgery; impact on fertility \\
requires further investigation [31, 41, 46] \\
Rltrasound-guided, high-intensity- \\
$\begin{array}{c}\text { Reduces fibroid symptoms and decreases fibroid and uterine volume with no reported permanent adverse } \\
\text { foffects [61] }\end{array}$ \\
$\begin{array}{c}\text { Transcervical radiofrequency ablation } \\
\text { Minimally invasive; uses radiofrequency energy to ablate fibroids; not yet included in treatment guide- } \\
\text { lines [62] }\end{array}$ \\
\hline
\end{tabular}

MRI, magnetic resonance imaging

\section{Conclusions}

When considering treatment plans for patients with uterine fibroids, important first steps include recognition, acknowledgment, and validation of symptoms; appropriate investigations (including imaging) to diagnose and classify specific fibroid subtypes; and consideration of individual patient needs (including effects on QOL or access to specific interventions) to guide management. This callto-action highlights challenges and limitations associated with uterine fibroid management; discusses newer options, based on the best available evidence, currently available for the medical management of uterine fibroids; and serves as a guide for physicians to select the appropriate treatment based on individual patient characteristics and needs. Medical options and minimally invasive treatments are generally recommended as first steps for the treatment of uterine fibroids, with other surgical options available if symptoms persist or if the patient prefers surgery as firstline treatment.

Acknowledgements Medical writing assistance, funded by AbbVie, was provided by Callie A S Corsa, PhD, and Kersten Reich, MPH, CMPPTM, of JB Ashtin. AbbVie Inc. reviewed a final version, but did not participate in the design, writing, or approval of this publication.

Author Contribution All authors equally contributed to the development, review, and approval of this manuscript, and in the decision to submit this review to Reproductive Sciences.

Funding AbbVie Inc. provided funding for this manuscript and approved the final version, but did not participate in the design, writing, or review of this report

Data Availability Not applicable.

Code Availability Not applicable.

\section{Declarations}

Ethics Approval Not applicable.

Consent to Participate Not applicable.

Consent for Publication Not applicable.

Conflict of Interest N.L. has received funding/grant support from AbbVie and Bayer, and honorarium for consultancy from AbbVie, Baxter, Bayer, and TerSera. M.L. has received funding/grant support from AbbVie and the Australian Woman and Children's Foundation, and honorarium for consultancy from Bayer, GE Healthcare, and TerSera. A.M. has received funding/grant support from $\mathrm{AbbVie}$, and honorarium for consultancy from AbbVie, Bayer, and Pfizer. S.S. has received funding/grant support from AbbVie, Allergan, and Bayer, and honorarium for consultancy from AbbVie, Allergan, Bayer, Myovant, and TerSera. A.A-H. has received funding/grant support from the National Institute of Health and Crila, and honorarium for consultancy from AbbVie, Allergan, Bayer, Myovant, Novartis, and OBS-EVA. L.B. has received royalties from Elsevier, UpToDate, and Wolter Kluwers.

Open Access This article is licensed under a Creative Commons Attribution 4.0 International License, which permits use, sharing, adaptation, distribution and reproduction in any medium or format, as long as you give appropriate credit to the original author(s) and the source, provide a link to the Creative Commons licence, and indicate if changes were made. The images or other third party material in this article are included in the article's Creative Commons licence, unless indicated otherwise in a credit line to the material. If material is not included in the article's Creative Commons licence and your intended use is not permitted by statutory regulation or exceeds the permitted use, you will need to obtain permission directly from the copyright holder. To view a copy of this licence, visit http://creativecommons.org/licenses/by/4.0/. 


\section{References}

1. Bourne T, Leonardi M, Kyriacou C, et al. ISUOG consensus statement on rationalization of gynecological ultrasound services in context of SARS-CoV-2. Ultrasound Obstet Gynecol. 2020;55:879-85.

2. American College of Obstetricians and Gynecologists. Management of symptomatic uterine leiomyomas: ACOG practice bulletin, number 228. Obstet Gynecol. 2021;137:e100-15.

3. Stewart EA. Clinical practice. Uterine fibroids N Engl J Med. 2015;372:1646-55.

4. Baird DD, Dunson DB, Hill MC, et al. High cumulative incidence of uterine leiomyoma in black and white women: ultrasound evidence. Am J Obstet Gynecol. 2003;188:100-7.

5. Go VAA, Thomas MC, Singh B, et al. A systematic review of the psychosocial impact of fibroids before and after treatment. Am J Obstet Gynecol. 2020;223:674-708 e8.

6. Cardozo ER, Clark AD, Banks NK, et al. The estimated annual cost of uterine leiomyomata in the United States. Am J Obstet Gynecol. 2012;206(211):e1-9.

7. Hartmann KE, Fonnesbeck C, Surawicz T, et al. Management of uterine fibroids. AHRQ comparative effectiveness review. Rockville, MD. 2017. Available at: https://effectivehealthcare.ahrq.gov/ sites/default/files/pdf/cer-195-uterine-fibroids-final-revision.pdf. Accessed 05 May 2021.

8. Marshall LM, Spiegelman D, Barbieri RL, et al. Variation in the incidence of uterine leiomyoma among premenopausal women by age and race. Obstet Gynecol. 1997;90:967-73.

9. Eltoukhi HM, Modi MN, Weston M, et al. The health disparities of uterine fibroid tumors for African American women: a public health issue. Am J Obstet Gynecol. 2014;210:194-9.

10. Marsh EE, Al-Hendy A, Kappus D, et al. Burden, prevalence, and treatment of uterine fibroids: a survey of U.S. women. J Womens Health (Larchmt). 2018;27:1359-67.

11. Van den Bosch T, Dueholm M, Leone FP, et al. Terms, definitions and measurements to describe sonographic features of myometrium and uterine masses: a consensus opinion from the Morphological Uterus Sonographic Assessment (MUSA) group. Ultrasound Obstet Gynecol. 2015;46:284-98.

12. Bougie O, Bedaiwy MA, Laberge $\mathrm{P}$, et al. Quality of ultrasonography reporting and factors associated with selection of imaging modality for uterine fibroids in Canada: results from a prospective cohort registry. CMAJ Open. 2020;8:E506-13.

13. Zhang M, Wasnik AP, Masch WR, et al. Transvaginal ultrasound shear wave elastography for the evaluation of benign uterine pathologies: a prospective pilot study. J Ultrasound Med. 2019;38:149-55.

14. Testa AC, Di Legge A, Bonatti M, et al. Imaging techniques for evaluation of uterine myomas. Best Pract Res Clin Obstet Gynaecol. 2016;34:37-53.

15. Gorgulu FF, Okcu NT. Which imaging method is better for the differentiation of adenomyosis and uterine fibroids? J Gynecol Obstet Hum Reprod. 2021;50:102002.

16. Munro MG, Critchley HO, Broder MS, et al. FIGO classification system (PALM-COEIN) for causes of abnormal uterine bleeding in nongravid women of reproductive age. Int J Gynaecol Obstet. 2011;113:3-13.

17. Munro MG, Critchley HOD, Fraser IS, et al. The two FIGO systems for normal and abnormal uterine bleeding symptoms and classification of causes of abnormal uterine bleeding in the reproductive years: 2018 revisions. Int J Gynaecol Obstet. 2018;143:393-408.

18. Commandeur AE, Styer AK, Teixeira JM. Epidemiological and genetic clues for molecular mechanisms involved in uterine leiomyoma development and growth. Hum Reprod Update. 2015;21:593-615.

19. Zhang G, Yu X, Zhu L, et al. Preoperative clinical characteristics scoring system for differentiating uterine leiomyosarcoma from fibroid. BMC Cancer. 2020;20:514.

20. Chevallard M, Belloli L, Ughi N, et al. Use of telemedicine during the COVID-19 pandemic in patients with inflammatory arthritis: a retrospective study on feasibility and impact on patient-reported outcomes in a real-life setting. Rheumatol Int. 2021;41:1253-61.

21. Walia B, Shridhar A, Arasu P, et al. US physicians' perspective on the sudden shift to telehealth: survey study. JMIR Hum Factors. 2021;8:e26336.

22. Yuan N, Pevnick JM, Botting PG, et al. Patient use and clinical practice patterns of remote cardiology clinic visits in the era of COVID-19. JAMA Netw Open. 2021;4:e214157.

23. Strong SM, Magama Z, Mallick R, et al. Waiting for myomectomy during the COVID-19 pandemic: the vicious cycle of psychological and physical trauma associated with increased wait times. Int J Gynaecol Obstet. 2020;151:303-5.

24. Saha S, Roy KK, Zangmo R, et al. Gynecological laparoscopic surgeries in the era of COVID-19 pandemic: a prospective study. Obstet Gynecol Sci. 2021;64:383-9.

25. Carugno J, Di Spiezio Sardo A, Alonso L, et al. COVID-19 pandemic. Impact on hysteroscopic procedures: a consensus statement from the Global Congress of Hysteroscopy Scientific Committee. J Minim Invasive Gynecol. 2020;27:988-92.

26. Brown KM, Robinson GE, Nadelson CC, et al. Psychological impact of COVID-19 on minority women. J Nerv Ment Dis. 2021;209:695-6.

27. Little $\mathrm{C}$, Alsen $\mathrm{M}$, Barlow $\mathrm{J}$, et al. The impact of socioeconomic status on the clinical outcomes of COVID-19; a retrospective cohort study. J Community Health. 2021;46:794-802.

28. Batty GD, Gaye B, Gale CR, et al. Explaining ethnic differentials in COVID-19 mortality: cohort study. Am J Epidemiol. 2021.

29. Khanna N, Klyushnenkova EN, Kaysin A. Association of COVID19 with race and socioeconomic factors in family medicine. J Am Board Fam Med. 2021;34:S40-7.

30. Al-Hendy A, Myers ER, Stewart E. Uterine fibroids: burden and unmet medical need. Semin Reprod Med. 2017;35:473-80.

31. Giuliani E, As-Sanie S, Marsh EE. Epidemiology and management of uterine fibroids. Int J Gynaecol Obstet. 2020;149:3-9.

32. Nelson AL, Ritchie JJ. Severe anemia from heavy menstrual bleeding requires heightened attention. Am J Obstet Gynecol. 2015;213(97):e1-6.

33. Vilos GA, Allaire C, Laberge PY, et al. The management of uterine leiomyomas. J Obstet Gynaecol Can. 2015;37:157-78.

34. Recommendations to prevent and control iron deficiency in the United States. Centers for Disease Control and Prevention. MMWR Recomm Rep. 1998;47:1-29.

35. Favrat B, Balck K, Breymann C, et al. Evaluation of a single dose of ferric carboxymaltose in fatigued, iron-deficient womenPREFER a randomized, placebo-controlled study. PLoS One. 2014;9:e94217.

36. Wenger MJ, Rhoten SE, Murray-Kolb LE, et al. Changes in iron status are related to changes in brain activity and behavior in Rwandan female university students: results from a randomized controlled efficacy trial involving iron-biofortified beans. J Nutr. 2019;149:687-97.

37. Friedman AJ, Chen Z, Ford P, et al. Iron deficiency anemia in women across the life span. J Womens Health (Larchmt). 2012;21:1282-9.

38. Murji A, Lam M, Allen B, et al. Risks of preoperative anemia in women undergoing elective hysterectomy and myomectomy. Am J Obstet Gynecol. 2019;221:629e1-e18. 
39. Muñoz M, Acheson AG, Auerbach M, et al. International consensus statement on the peri-operative management of anaemia and iron deficiency. Anaesthesia. 2017;72:233-47.

40. Farris M, Bastianelli C, Rosato E, et al. Uterine fibroids: an update on current and emerging medical treatment options. Ther Clin Risk Manag. 2019;15:157-78.

41. De La Cruz MS, Buchanan EM. Uterine fibroids: diagnosis and treatment. Am Fam Physician. 2017;95:100-7.

42. Donnez J, Dolmans MM. Hormone therapy for intramural myomarelated infertility from ulipristal acetate to $\mathrm{GnRH}$ antagonist: a review. Reprod Biomed Online. 2020;41:431-42.

43. Clarke-Pearson DL, Geller EJ. Complications of hysterectomy. Obstet Gynecol. 2013;121:654-73.

44. Beyan E, Inan AH, Emirdar V, et al. Comparison of the effects of total laparoscopic hysterectomy and total abdominal hysterectomy on sexual function and quality of life. Biomed Res Int. 2020;2020:8247207.

45. Laberge PY, Murji A, Vilos GA, et al. Guideline no. 389-medical management of symptomatic uterine leiomyomas - an addendum. J Obstet Gynaecol Can. 2019;41:1521-4.

46. Mas A, Tarazona M, Dasi Carrasco J, et al. Updated approaches for management of uterine fibroids. Int $\mathrm{J}$ Womens Health. 2017;9:607-17.

47. Bartels CB, Cayton KC, Chuong FS, et al. An evidence-based approach to the medical management of fibroids: a systematic review. Clin Obstet Gynecol. 2016;59:30-52.

48. Jiang W, Shen Q, Chen M, et al. Levonorgestrel-releasing intrauterine system use in premenopausal women with symptomatic uterine leiomyoma: a systematic review. Steroids. 2014;86:69-78.

49. Schlaff WD, Ackerman RT, Al-Hendy A, et al. Elagolix for heavy menstrual bleeding in women with uterine fibroids. N Engl J Med. 2020;382:328-40.

50. Ali M, A RS, Al Hendy A. Elagolix in the treatment of heavy menstrual bleeding associated with uterine fibroids in premenopausal women. Expert Rev Clin Pharmacol. 2021;14:427-37.

51. Al-Hendy A, Lukes AS, Poindexter AN 3rd, et al. Treatment of uterine fibroid symptoms with relugolix combination therapy. $\mathrm{N}$ Engl J Med. 2021;384:630-42.
52. Donnez J, Taylor HS, Taylor RN, et al. Treatment of endometriosis-associated pain with linzagolix, an oral gonadotropin-releasing hormone-antagonist: a randomized clinical trial. Fertil Steril. 2020;114:44-55.

53. Lethaby A, Puscasiu L, Vollenhoven B. Preoperative medical therapy before surgery for uterine fibroids. Cochrane Database Syst Rev. 2017;11:CD000547.

54. Murji A, Whitaker L, Chow TL, et al. Selective progesterone receptor modulators (SPRMs) for uterine fibroids. Cochrane Database Syst Rev. 2017;4:CD010770.

55. Middelkoop MA, Bet PM, Drenth JPH, et al. Risk-efficacy balance of ulipristal acetate compared to surgical alternatives. Br J Clin Pharmacol. 2020.

56. Shah N, Egbase E, Sideris M, et al. What happens after randomised controlled trials? Uterine fibroids and ulipristal acetate: systematic review and meta-analysis of "real-world" data. Arch Gynecol Obstet. 2021;303:1121-30.

57. Ekanem E, Talaulikar V. Medical therapy for fibroids: what next for ulipristal acetate? Adv Ther. 2021;38:137-48.

58. Ciebiera M, Ali M, Prince $\mathrm{L}$, et al. The evolving role of natural compounds in the medical treatment of uterine fibroids. J Clin Med. 2020;9.

59. Porcaro G, Angelozzi P. Uterine fibroid treatment with vitamin D combined with epigallocatechin gallate and vitamin B6: a controlled pilot study. IJMDAT. 2021;4:e300.

60. Cope AG, Young RJ, Stewart EA. Non-extirpative treatments for uterine myomas: measuring success. J Minim Invasive Gynecol. 2021;28:442-52 e4.

61. Lee JS, Hong GY, Lee KH, et al. Safety and efficacy of ultrasound-guided high-intensity focused ultrasound treatment for uterine fibroids and adenomyosis. Ultrasound Med Biol. 2019;45:3214-21.

62. Christoffel L, Romer T, Schiermeier S. Transcervical radiofrequency ablation of uterine fibroids global registry (SAGE): study protocol and preliminary results. Med Devices (Auckl). 2021;14:77-84. 